\title{
Formato e área de comedouros em tanques-rede para juvenis de tilápia do Nilo
}

\author{
Marengoni, N.G. ${ }^{1} ;$; Mahl, I. ${ }^{2}$; Albuquerque, D.M. ${ }^{3}$; Moura, M.C. ${ }^{4}$; Sanches, E.A. ${ }^{5}$ e Piana, P.A. ${ }^{6}$
}

\author{
'Centro de Ciências Agrárias. Curso de Zootecnia. Universidade Estadual do Oeste do Paraná. Marechal Cândido Rondon, PR. Brasil. \\ ${ }^{2}$ Cooperativa Agroindustrial Consolata-Copacol. Nova Aurora. Brasil. \\ ${ }^{3}$ Faculdade de Ciências Agrárias. Universidade Federal da Grande Dourados-UFGD. Dourados. Brasil. \\ 4Pontifícia Universidade Católica do Paraná-PUC. Toledo. Brasil. \\ ${ }^{5}$ Universidade Estadual Paulista Júlio de Mesquita Filho-Unesp. Registro. Brasil. \\ ${ }^{\circ}$ Centro de Engenharias e Ciências Exatas. Universidade Estadual do Oeste do Paraná. Toledo. Brasil.
}

\section{PaLAVRAS-ChAVE ADICIONAIS}

Desempenho produtivo.

Homogeneidade do plantel.

Índices corporais.

Oreochromis niloticus.

Tilapicultura.

\section{RESUMO}

Objetivou-se avaliar formatos e áreas de comedouros utilizados em tanques-rede sobre o desempenho, homogeneidade dos lotes e índices corporais de tilápia do Nilo. Foram utilizados 2.700 juvenis de tilápia com peso médio de 5,95 $\pm 1,23 \mathrm{~g}$, distribuídos em 27 tanques-rede 10,5 $\times 0,5 \times 1,0 \mathrm{~m})$, em um delineamento experimental em esquema fatorial, com dois formatos de comedouros (quadrado e circular) e quatro áreas $(100,75,50$ ou $25 \%$ ) e um tratamento controle (isento de comedouro), com três repeticões. Após 64 dias avaliaram-se os parâmetros zootécnicos de ganho em peso, comprimento total, taxa de crescimento específico, sobrevivência, conversão alimentar aparente e homogeneidade dos lotes. Os índices viscerossomático, hepatossomático e gordura visceral também foram avaliados. O formato dos alimentadores não influenciou ( $p>0,05)$ os parâmetros de desempenho de juvenis de tilápia, no entanto, a ausência de comedouros afetou $(p<0,05)$ negativamente a sobrevivência. Observaram-se menores valores de ganho em peso $(25,45 \mathrm{~g})$ e comprimento total $(11,50 \mathrm{~cm})$ e conversão alimentar aparente superior dos peixes cultivados nos tanques-rede isentos de comedouros. $O$ formato e a área dos comedouros não influenciaram $(p>0,05)$ nos índices corporais, entretanto, os peixes submetidos aos tanques-rede sem comedouros apresentaram menores $(p<0,05)$ índices hepatossomático, viscerossomático e de gordura visceral. As diferentes áreas e formatos de comedouro não interferem no desempenho produtivo na fase juvenil de tilápia do Nilo em tanques-rede de pequeno volume. Considerando um melhor aproveitamento das dietas no desempenho das tilápias, recomenda-se a utilização de pelo menos uma das combinações entre formato e área de comedouro utilizado na fase cultivada.

\section{Format and size of feeders in cages for Nile tilapia juvenile}

\section{SUMMARY}

\section{ADDITIONAL KEYWORDS}

Productive performance.

Homogeneity of lots.

Body indexes.

Oreochromis niloticus.

Tilapiculture.

INFORMATION

Cronología del artículo.

Recibido/Received: 22.06 .2016

Aceptado/Accepted: 20.09.2017

On-line: 15.10.2017

Correspondencia a los autores/Contact e-mail:

ngmget@hotmail.com

\section{INTRODUÇÃO}

A aquicultura em águas continentais é uma atividade agropecuária promissora, na qual, a tilápia do Nilo é a espécie mais cultivada no Brasil representando aproximadamente $45 \%$ do total da produção
This study aimed to evaluate formats and areas of feeders used in cages on production performance, uniformity of lots and body indexes of Nile tilapia. 2700 juvenile tilapia with mean weight of $5.95 \pm 1.23 \mathrm{~g}$ were used, distributed in 27 cages $(0.5 \times 0.5 \times 1.0 \mathrm{~m})$ in a completely randomized design in a factorial model, consisting of feeders with two formats (square and circular) and four areas $(100,75,50$ or $25 \%$ ) and a control (feeder free) with three replicates. After 64 days, we evaluated the performance parameters of weight gain, total length, specific growth rate, survival, feed conversion and uniformity of lots. The viscerosomatic, hepatosomatic and visceral fat indexes were also evaluated. The format of feeders did not influence $(p>0.05)$ the performance parameters of tilapia juvenile, however, the lack of a feeder area affected $(p<0.05)$ negatively the survival of the juvenile. We observed lower values of weight gain $(25.45 \mathrm{~g})$ and length $(11.50 \mathrm{~cm})$ and higher feed conversion for fish reared in cages at absent of feeders. The feed conversion was higher $(p<0.05)$ for fish in cages without feeder compared to the others. The format and the area of feeders did not affect $(p>0.05)$ corporeal indexes, however, fish subjected to cages without feeders had lower $(p<0.05)$ hepatosomatic, viscerosomatic, and visceral fat indexes. The different areas and formats feeder do not interfere in the productive performance in the juvenile phase of Nile tilapia in cages of small volume. For a better utilization of diets on the fish performance, we recomend to use, at least, one of the combinations of size and format of feeder used during cultivation in cages. 
restrição da legislação ambiental (Barroso et al., 2015). No estado do Paraná o cultivo de grãos (soja, milho e trigo) é predominante na produção agropecuária, mas a cadeia produtiva da tilápia é desenvolvida como uma fonte complementar de renda para o produtor rural, e o Estado já figura como um dos principais polos produtores da espécie no País (Marengoni et al., 2007).

Na produção iniciada com alevinos de tilápia do Nilo (Oreochromis niloticus) é comum ao final do cultivo lotes de peixes apresentarem maior variação de tamanho e peso devido às características territorialistas e hierárquicas da espécie (Merighe et al., 2004). Este efeito negativo pode ser minimizado em ambientes com menor estresse e competição pelo alimento, melhorando a uniformidade dos lotes (Rebouças et al., 2014). Além disso, uma estratégia encontrada pelos aquicultores é a utilização de peixes na fase juvenil, especialmente quando os exemplares de tilápia são destinados ao sistema de cultivo em tanques-rede (Moraes et al., 2009). Na fase juvenil, os peixes apresentam baixa mortalidade, facilidade de seleção e classificação, dando origem a plantéis com maior uniformidade o que possibilita melhor controle e planejamento da produção.

Outro aspecto importante de um sistema de criação são as práticas de manejo e alimentação, que associadas com outros fatores, influenciam na dinâmica da qualidade da água. Algumas alterações provocadas pela criação de peixes são caracterizadas pela aceleração do processo de eutrofização da água dos viveiros. Desta forma, os manejos praticados no cotidiano da criação piscícola merecem atenção especial, como a utilização de comedouros na produção em tanques-rede objetivando a retenção e melhor aproveitamento da ração pelos peixes (Sandoval Júnior et al., 2013). A utilização do sistema de arraçoamento adequado possibilita otimizar o aproveitamento de nutrientes e o crescimento dos animais, além de reduzir a liberação de poluentes no meio aquático (Cyrino et al., 2010; Wild et al., 2014).

Uma forma de avaliar o bem-estar dos peixes do ponto de vista nutricional num sistema de criação, é estimar o fator de condição dos animais, a relação entre o seu peso e comprimento (Gomiero e Braga, 2005). Adicionalmente, os índices hepatossomático, viscerossomático e de gordura visceral são igualmente importantes, pois sugerem alterações morfológicas ou fisiológicas em resposta aos diferentes manejos alimentares praticados, podendo ser adotados como informações complementares aos dados biométricos utilizados nas atividades aquícolas (Tavares-Dias et al., 2000, 2008; Bombardelli et al., 2010). Estudos de diferentes formatos e áreas de comedouros utilizados em tanques-rede para o cultivo de tilápia do Nilo ainda parecem ser escassos e incipientes para fomentar a produção de juvenis. Desta forma, objetivou-se avaliar as possíveis influências de área e do formato de comedouros instalados em tanques-rede, sobre o desempenho zootécnico, homogeneidade dos lotes e índices corporais de juvenis de tilápia do Nilo da linhagem GIFT.

\section{MATERIAL E MÉTODOS}

O experimento foi conduzido no Instituto de Pesquisa e Aquicultura Ambiental (InPAA) em Toledo, Paraná, Brasil, durante 64 dias. Foram utilizados 2700 juvenis de tilápia do Nilo da linhagem GIFT (Genetically Improved Farmed Tilapia), sexualmente invertidos para machos, com peso inicial de 5,95 $\pm 1,23 \mathrm{~g}$, e tamanho inicial de 6,79 $\pm 0,44 \mathrm{~cm}$, adquiridos de piscicultura comercial. Os juvenis foram distribuídos em 27 tanques-rede $(0,5 \times 0,5 \times 1,0 \mathrm{~m})$ com volume útil de $0,2 \mathrm{~m}^{3}$, malha $4,0 \mathrm{~mm}$, material Sannet ${ }^{\circledR}$, instaladas em um viveiro de $800 \mathrm{~m}^{2}$ com paredes revestidas de alvenaria e fundo de solo natural.

Foi utilizado o delineamento experimental inteiramente casualizado em esquema fatorial $2 \times 4$ com três repetições. Os cultivos foram compostos por dois formatos de comedouros (quadrado ou circular), quatro áreas (100\%; $75 \% ; 50 \%$ ou $25 \%)$ e um cultivo controle, isento de comedouro. Os comedouros foram confeccionados utilizando tela de malha Sannet ${ }^{\circledR}(2 \mathrm{~mm})$ fixados a uma moldura de tubulação de PVC, ajustados no formato e área utilizada. Os comedouros possuíam altura de 40 $\mathrm{cm}$ e foram fixados no centro dos tanques-rede, de modo que a borda superior e inferior dos comedouros ocupasse respectivamente 15 e $25 \mathrm{~cm}$ acima e abaixo da linha da água (Kubitza, 2011; Sandoval Júnior et al., 2013).

Considerou-se uma unidade experimental um tanque-rede contendo 100 peixes. As áreas dos comedouros foram calculadas a partir do maior comedouro circular, sendo este caracterizado com área de $100 \%$. Os demais foram calculados de modo que os dois formatos apresentassem a mesma área conforme os cultivos. O comedouro foi confeccionado com o mesmo material dos tanques-rede, entretanto, com malha de $2 \mathrm{~mm}$ de espaçamento a fim de conter a ração fornecida.

A temperatura da água foi monitorada uma vez ao dia, sempre no final da tarde após a última alimentação. Semanalmente foram mensuradas alguns parâmetros físicos e químicos da água, como oxigênio dissolvido com oxímetro (YSI 550A), pH com auxílio de potenciômetro (HI 8314, Hanna Instruments ${ }^{\circledR}$ ) e condutividade com condutivímetro (HI 9033, Hanna Instruments ${ }^{\circledR}$ ). As mensurações das variáveis foram praticadas no fundo e na superfície da coluna de água da entrada, meio e saída de água do viveiro. A transparência foi monitorada com disco de Secchi, também semanalmente na entrada, meio e saída de água do viveiro.

Realizou-se o manejo alimentar manual com ração comercial Nutron ${ }^{\circledR}$ extrusada de $2 \mathrm{~mm}$, contendo $36 \%$ de proteína bruta, $8 \%$ de extrato etéreo, 
$6 \%$ de fibra bruta, $13 \%$ de matéria mineral, 1,5 de cálcio (máximo), 0,5 de fósforo (máximo), além de premix vitamínico e mineral. A frequência de arraçoamento foi fixada em quatro vezes ao dia nos horários 8, 11, 14 e $17 \mathrm{~h}$. A quantidade de ração fornecida foi estimada em função da biomassa total de cada tanque-rede e ajustada quinzenalmente por meio de biometrias de todos os peixes das unidades experimentais, conforme adaptações e recomendações técnicas do fornecedor. Foram oferecidos $10 \%$ da biomassa nos primeiros 15 dias de experimento, $8 \%$ entre $15^{\circ}$ e $30^{\circ}, 5,5 \%$ entre os $30^{\circ}$ e $45^{\circ}$ e $4,5 \%$ no $45^{\circ}$ a $64^{\circ}$ dias.

Ao final do período experimental realizou-se a pesagem e contagem de todos os peixes de cada tanque-rede a fim de avaliar o peso final, ganho em peso, conversão alimentar aparente e sobrevivência. De cada unidade experimental foram coletados aleatoriamente 30 juvenis para avaliação do peso e comprimento total e medida do peso do fígado, gordura visceral (sem o fígado) e vísceras totais (vísceras e fígado) a fim de determinar os índices hepatossomático, de gordura visceral e viscerossomático, respectivamente.

Com o intuito de avaliar a homogeneidade do lote, foi determinado o desvio padrão do peso final, do comprimento final e do fator de condição em 30 peixes de cada unidade experimental. A taxa de crescimento específico (TCE) e o fator de condição de Fulton, chamado isométrico $(\mathrm{Kn})$ foram calculados por meio das equações:

TCE $=\left\{\left[\left(\ln P_{\mathrm{f}}-\ln \mathrm{P}_{\mathrm{i}}\right) / \mathrm{n}^{\circ}\right.\right.$ de dias $\left.] \times 100\right\} ; \mathrm{e} \mathrm{Kn}$ $=\left[\left(\right.\right.$ peso corporal $/$ comprimento corporal $\left.\left.{ }^{3}\right) \times 100\right]$; em que, ln é logaritmo natural; $\mathrm{P}_{\mathrm{f}}$ peso final; e $\mathrm{P}_{\mathrm{i}}$, peso inicial.

Os valores dos parâmetros de qualidade de água, $\mathrm{pH}$, condutividade, oxigênio dissolvido, transparência e temperatura da água, e conversão alimentar aparente e temperatura da água foram agrupados por quinzenas ao longo do experimento e submetidos à análise de correlação linear de Pearson a 5\% de probabilidade (Rodrigues, 2002).

Para avaliar o efeito do formato e da área dos alimentadores sobre peso final (PF), desvio padrão do PF (DPPF), ganho em peso (GP), comprimento total final $(\mathrm{CF})$, desvio padrão do $\mathrm{CF}$ (DPCF), fator de condição (Kn), desvio padrão do Kn (DPKn), taxa de crescimento específico (TCE), sobrevivência (SO) e conversão alimentar aparente (CA); índice viscerossomático (IVS), hepatossomático (IHS) e gordura visceral (IGV), foram empregadas análises de variância bifatorial seguidas do teste de comparação de médias de Tukey, a 5\% de probabilidade (Rodrigues, 2002), conforme a componente sistemática:
$\mathrm{VR}_{\mathrm{ijk}}=\mu+\alpha_{\mathrm{i}} \times$ Formato $_{\mathrm{i}}+\beta_{\mathrm{j}} \times$ Área $_{\mathrm{j}}+\chi_{\mathrm{ij}} \times$ Formato $_{\mathrm{i}} \times$ Área $_{\mathrm{j}}+\varepsilon_{\mathrm{ijk}}$

onde:

$\mathrm{VR}_{\mathrm{ijk}}=$ Variável resposta (índices zootécnicos e índices corporais);

Formato $=$ Fator "Formato de comedouro" $(\mathrm{i}=$ circular ou quadrado);

Área = Fator "Área do comedouro" ( $j=25,50$, 75 ou $100 \%)$;

$\mathrm{k}=$ número de réplicas (3);

$\mu, \alpha, \beta$ e $\chi=$ parâmetros do modelo;

$\varepsilon=$ resíduo com distribuição normal e variância homogênea.

Para verificar se a utilização de comedouros de diferentes formatos e áreas foi eficiente, empregou-se a análise de variância unifatorial sobre os diferentes tratamentos, seguido do teste de Dunnett para comparação das médias com o controle, a $5 \%$ de probabilidade (Rodrigues, 2002), conforme a componente sistemática:

$\mathrm{VR}_{\mathrm{ijk}}=\mu+\alpha_{\mathrm{i}} \times$ Cultivo $_{\mathrm{i}}+\varepsilon_{\mathrm{ik}} ;$

no qual:

$\mathrm{VR}_{\mathrm{ijk}}=$ Variável resposta (índices zootécnicos e índices corporais);

Área ${ }_{\mathrm{i}}=$ sem comedouro, circular de área 25\%, circular área 50\%, circular área 75\%, circular área $100 \%$, quadrado área $25 \%$, quadrado área 50\%, quadrado área $75 \%$, quadrado área $100 \%$;

$\mathrm{k}$ = número de réplicas (3);

$\mu$ e $\alpha$ = parâmetros do modelo;

$\varepsilon=$ resíduo com distribuição normal e variância homogênea.

Os procedimentos estatísticos foram realizados com o auxílio do software Statistica 7.0, sob o domínio da Universidade Estadual do Oeste do Paraná, conforme a licença de utilização número 0607a. Os ensaios e procedimentos foram realizados de acordo com os princípios propostos pela Sociedade Brasileira de Ciência em Animais de Laboratório/Colégio Brasileiro de Experimentação Animal (SBCAL/COBEA) e foram aprovados pelo Comitê de Ética em Experimentação Animal e Aulas Práticas da Universidade Estadual do Oeste do Paraná (protocolo no 81 / 2009 CEEAAP / Unioeste).

\section{RESULTADOS E DISCUSSÃO}

Em relação aos parâmetros de qualidade da água analisados durante o período experimental, foram encontrados os valores (média \pm desvio padrão) de 7,57 \pm 


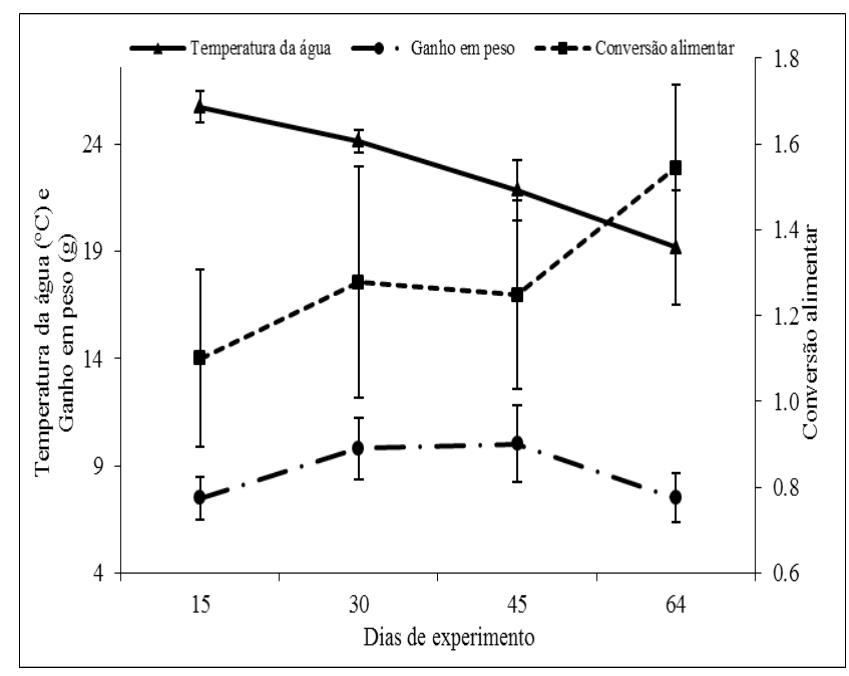

Figura 1. Valores (média \pm desvio padrão) para temperatura da água, conversão alimentar e ganho em peso durante o experimento com alevinos de tilápia do Nilo da linhagem GIFT (Mean values \pm standard deviation of water temperature, feed conversion and weight gain of experimental Nile tilapia fingerlings).

0,49 para $\mathrm{pH} ; 49,56 \pm 8,78 \mu \mathrm{S} \mathrm{cm} \mathrm{cm}^{-1}$ para condutividade; $5,15 \pm 1,34 \mathrm{mg} \mathrm{L}^{-1}$ para oxigênio dissolvido e 1,17 \pm $0,3 \mathrm{~m}$ para transparência. Ocorreram flutuações nas concentrações de oxigênio dissolvido e condutividade, e estabilidade nos valores de $\mathrm{pH}$, possivelmente devido à dinâmica do viveiro. Apesar das variações, os parâmetros mantiveram-se nas condições normais e adequadas aos limites de conforto para criação de tilápia do Nilo (Boyd e Tucker, 1998; Boyd, 2005; ElSayed, 2006).

A análise de correlação de Pearson revelou correlação $(p<0,05)$ diretamente proporcional para temperatura e condutividade $(r=0,8360)$ e correlação inversamente proporcional entre temperatura e oxigênio dissolvido $(\mathrm{r}=-0,6903)$; temperatura e transparência $(\mathrm{r}=$ $-0,8361)$ e condutividade e transparência $(r=-0,7506)$. Verificou-se correlação negativa $(p<0,05)$ entre a temperatura e o oxigênio dissolvido, possivelmente devido a maior capacidade de retenção de oxigênio em baixas temperaturas (Boyd e Tucker, 1998). A temperatura da água variou entre 14 a $27^{\circ} \mathrm{C}$, com média de $22,25 \pm$ $3,08^{\circ} \mathrm{C}$, ficando abaixo da temperatura ideal indicada para o cultivo de tilápia do Nilo que é de $28^{\circ} \mathrm{C}$ (ElSayed, 2006). Este fator proporcionou a diminuição no consumo de ração, principalmente nos últimos dias do experimento (figura 1). As oscilações no desempenho de tilápia do Nilo, relacionadas por declínios na temperatura da água de cultivo, também foram observadas por Rezk et al. (2002) e Marengoni et al. (2008).

Houve um crescente ganho em peso entre o $15^{\circ} \mathrm{e}$ o $30^{\circ}$ dia e uma estabilização entre o $30^{\circ}$ e o $45^{\circ}$ dia. Observou-se uma diminuição no ganho em peso à medida que a temperatura diminuiu, pois este parâmetro da água é um fator limitante ao crescimento dos peixes. Consequentemente o índice de conversão alimentar elevou-se entre o $45^{\circ}$ e $64^{\circ}$ dia. Esta hipótese é corroborada pela correlação inversamente proporcional observada entre a temperatura e a conversão alimentar aparente (figura 1) $\left(r^{2}=0,4324 ; r=-0,6576 ; p=\right.$ 0,$001 ; \mathrm{CAA}=2,7283-0,0634 \times \mathrm{Temp}$ ), indicando que a tilápia do Nilo converte melhor a ração em peso corporal quando a temperatura da água está próxima ao conforto térmico (Boyd e Tucker, 1998; El-Sayed, 2006).

$\mathrm{O}$ formato e a área dos comedouros não apresentaram efeito $(p>0,05)$ sobre o peso final, entretanto observou-se uma interação entre o formato e a área $(\mathrm{p}<0,05)$, com maiores valores nos tanques contendo comedouros quadrados com área de 50\% (tabela I). Formato de área dos comedouros com bases iguais apresentam maior superfície de contato com a movimentação da água e consequentemente melhor a renovação, permitindo a manutenção de uma qualidade de água melhor no interior dos tanques-rede (Kubitza, 2011; Sandoval Junior et al., 2013).

Comparando os tanques-rede que continham comedouros aos isentos, verificou-se que a não utilização desse aparato influenciou $(p<0,05)$ negativamente no peso final dos peixes. $\mathrm{O}$ formato e a área expressaram efeito interativo sobre o tamanho dos peixes, sendo que o cultivo que continha comedouros no formato quadrado com área de $50 \%$ apresentou resultado superior àquele de formato quadrado com área de $100 \%$. Resultados semelhantes foram observados por Neves et al. (2008) estudando o desempenho produtivo das linhagens Bouaké e Chitralada, que ao final de um cultivo de 125 dias obtiveram um crescimento final entre 11,04 e $12,06 \mathrm{~cm}$. Adicionalmente, o desempenho no cultivo composto de comedouros quadrados com área de $50 \%$ e formato circular com área correspondente a $100 \%$ foram superiores aos isentos de comedouros. As demais combinações entre formatos e áreas de comedouros não apresentaram diferenças significativas para o comprimento total dos peixes ao final do período experimental $(p>0,05)$. Da mesma forma, menores valores para o fator de condição também foram observados nos animais mantidos em tanques-rede isentos de comedouros $(\mathrm{p}<0,05)$. Entretanto, não foi observado efeito de interação $(p>0,05)$ para o formato e área sobre os valores do fator de condição dos peixes (tabela I). O fator de condição é um parâmetro importante para avaliar o crescimento e índice de massa corporal capaz de refletir as interações entre o ambiente e o peixe, o que pode indicar o bem-estar das espécies (Tavares-Dias et al., 2008).

Verificou-se que os formatos e áreas de comedouros não influenciaram no ganho em peso (GP) $(\mathrm{p}>0,05)$, porém os valores médios de GP dos peixes nos tanques-rede isentos de comedouros foram significativamente inferiores aos que continham comedouros (tabela II). Neste trabalho foram observados GP diário de 0,55 g $\mathrm{dia}^{-1}$ e peso médio final de $44,50 \mathrm{~g}$, resultados superiores aos encontrados por Marengoni et al. (2008), que observaram GP diário de 0,43 g dia ${ }^{-1}$ e PF de 40,49 g, 


\begin{tabular}{|c|c|c|c|c|c|c|}
\hline & PFM (a) & DPPF (a) & & DPCTF $(\mathrm{cm})$ & $\mathrm{Kn}$ & DPKn \\
\hline & $F=0,0001$ & $F=0,0469$ & $F=0,0552$ & $F=0,1347$ & $\mathrm{~F}=0,1079$ & $F=2,7504$ \\
\hline Formato & $p=0,9711$ & $p=0,8313$ & $p=0,8173$ & $p=0,7184$ & $p=0,7468$ & $\mathrm{p}=0,1167$ \\
\hline Circular (C) & 44,54 & 12,95 & 12,55 & 1,13 & 2,19 & 0,20 \\
\hline Quadrado (Q) & 44,50 & 12,77 & 12,53 & 1,16 & 2,19 & 0,16 \\
\hline Área (\%) & $\begin{array}{l}F=0,4790 \\
p=0,7011\end{array}$ & $\begin{array}{l}F=1,1778 \\
p=0,3493\end{array}$ & $\begin{array}{l}F=0,6453 \\
p=0,5971\end{array}$ & $\begin{array}{l}F=1,0984 \\
p=0,3786\end{array}$ & $\begin{array}{l}F=0,3214 \\
p=0,8098\end{array}$ & $\begin{array}{l}F=0,4740 \\
p=0,7047\end{array}$ \\
\hline 25 & 44,44 & 12,02 & 12,52 & 1,23 & 2,18 & 0,18 \\
\hline 50 & 45,19 & 13,08 & 12,59 & 1,11 & 2,20 & 0,17 \\
\hline 75 & 45,05 & 12,30 & 12,61 & 1,17 & 2,18 & 0,20 \\
\hline 100 & 43,40 & 14,03 & 12,43 & 1,08 & 2,19 & 0,16 \\
\hline Formato x área & $\begin{array}{l}F=5,4790 \\
p=0,0088\end{array}$ & $\begin{array}{l}F=0,5353 \\
p=0,6647\end{array}$ & $\begin{array}{l}F=6,4975 \\
p=0,0044\end{array}$ & $\begin{array}{l}F=1,8062 \\
p=0,1866\end{array}$ & $\begin{array}{l}F=1,1906 \\
p=0,3448\end{array}$ & $\begin{array}{l}F=1,2262 \\
p=0,3326\end{array}$ \\
\hline$C \times 25$ & $44,42^{\mathrm{abB}}$ & 13,80 & $12,52^{\mathrm{abB}}$ & 1,18 & $2,19^{A}$ & 0,18 \\
\hline$C \times 50$ & $42,04^{\mathrm{abB}}$ & 13,23 & $12,29^{a b B}$ & 1,21 & $2,18^{\mathrm{A}}$ & 0,19 \\
\hline$C \times 75$ & $44,69^{\mathrm{abB}}$ & 12,63 & $12,63^{\mathrm{abB}}$ & 1,08 & $2,16^{\mathrm{AB}}$ & 0,24 \\
\hline$C \times 100$ & $47,00^{\mathrm{abA}}$ & 12,13 & $12,76^{\mathrm{abA}}$ & 1,07 & $2,21^{A}$ & 0,17 \\
\hline$Q \times 25$ & $44,45^{\mathrm{abB}}$ & 14,25 & $12,53^{\mathrm{abB}}$ & 1,29 & $2,18^{A}$ & 0,19 \\
\hline$Q \times 50$ & $48,34^{\mathrm{aA}}$ & 11,37 & $12,89^{\mathrm{aA}}$ & 1,00 & $2,21^{\mathrm{A}}$ & 0,15 \\
\hline$Q \times 75$ & $45,42^{\mathrm{abA}}$ & 13,54 & $12,59^{a b B}$ & 1,26 & $2,20^{A}$ & 0,15 \\
\hline$Q \times 100$ & $39,78^{\text {bв }}$ & 11,91 & $12,10^{\mathrm{bB}}$ & 1,09 & $2,17^{\mathrm{A}}$ & 0,16 \\
\hline Controle ou Isento & $33,32^{\mathrm{B}}$ & 10,15 & $11,50^{\mathrm{B}}$ & 1,12 & $2,05^{\mathrm{B}}$ & 0,16 \\
\hline CV (\%) & 6,02 & 7,85 & 1,99 & 8,92 & 0,82 & 16,65 \\
\hline
\end{tabular}

Estatística F e p-valor são apresentados para cada fonte de variação. Coeficiente de variação (CV) entre os cultivos também é fornecido. "Letras minúsculas distintas nas colunas indicam diferenças significativas $(p<0,05)$ pelo teste de Tukey (comparação entre os cultivos) e letras maiúsculas distintas nas colunas indicam diferenças significativas $(p<0,05)$ pelo teste de Dunnett (comparação dos cultivos com o controle ou isento).

também utilizando a linhagem GIFT, porém os peixes cultivados em hapas com diferentes densidades.

O formato ou área dos comedouros não influenciou $(p>0,05)$ na taxa de crescimento específico, entretanto, apresentou efeito interativo entre as variáveis, com maiores valores $(p<0,05)$ para os tanques que continham comedouro quadrado com área de $50 \%$ em relação ao formato quadrado com área de $100 \%$. As taxas de crescimento dos peixes cultivados nos tanques-rede isentos de comedouros foram inferiores $(p<0,05)$ aos demais, exceto para os formatos circular e quadrado, respectivamente com áreas de 50\% e 100\% (tabela II). Tachibana et al. (2008) observaram resultados semelhantes para taxa de crescimento específico, quando estudaram a densidade de estocagem de pós-larvas de tilápia do Nilo durante a reversão sexual. Os autores enfatizaram que o crescimento dos peixes pode ser atribuído a diversos fatores, como por exemplo, o consumo de ração, alteração nos hormônios de crescimento e estresse devido às densidades de estocagem elevadas. Associados a estes fatores é importante acompanhar o crescimento dos peixes, porque é um parâmetro ecológico importante que pode servir como um indicador das condições de higidez dos indivíduos em relação às condições ambientais (Siangas et al., 2012).

Para a sobrevivência, houve efeito apenas da área $(\mathrm{p}<0,05)$, no qual o maior valor foi observado em tanques-rede com comedouro com 50\% de área. A menor sobrevivência foi observada nos tanques-rede contendo comedouros com áreas 25 e 75\% (tabela II). Este fato possivelmente deve-se ao comportamento agressivo e territorialista que pode ser observado no cultivo de tilápia da linhagem GIFT (Mamun et al., 2004). Ao contrário do presente estudo, Neumann et al. (2009), avaliando desempenho de três linhagens de tilápia do Nilo mantidas em condições ambientais não controladas, obtiveram baixas taxas de sobrevivência em função de canibalismo causado pela heterogeneidade dos lotes, variações de temperatura e manejos excessivos. Os valores de sobrevivência observados neste trabalho, em que apenas os tanques-rede estavam povoados, dis- 
cordam daqueles encontrados por Mainardes-Pinto et al. (2007), com sobrevivências inferiores, variando entre 63 e $89,5 \%$, em um cultivo utilizando tanques-rede em viveiros povoados com tilápias.

Apesar disso, a principal causa do comportamento destes resultados em relação à área ocupada pelos comedouros é desconhecida e sugerem-se estudos complementares para comprovar tal efeito. Os formatos e áreas de comedouros não influenciaram a conversão alimentar aparente, porém os tanques-rede isentos de comedouros apresentaram maior índice que os demais $(p<0,05)$. Reduzir os valores de conversão alimentar, sem influenciar negativamente o desempenho dos peixes, pode representar uma economia significativa no custo referente à alimentação, além de vantagem ambiental, pois resulta em menor aporte de nutrientes para o ambiente (Wild et al., 2014).

O formato e a área dos comedouros utilizados no manejo alimentar dos juvenis de tilápia do Nilo não influenciaram $(\mathrm{p}>0,05)$ os índices corporais, entretanto, os peixes submetidos aos tanques-rede sem comedouros apresentaram menores índices viscerossomático, hepatossomático e de gordura visceral $(p<0,05)$, com valores de 10,87; 2,36 e 2,33\%, respectivamente (tabela III). Os lotes de peixes dos tanques-rede que continham comedouros circulares ou quadrados com áreas de 50\% apresentaram maiores índices de gordura visceral em relação ao cultivo isento de comedouro. O índice hepatossomático dos peixes produzidos em qualquer uma das combinações de formatos e áreas, com exceção do formato quadrado com área de $25 \%$, apresentou valores superiores $(p<0,05)$ ao índice hepatossomático dos peixes cultivados sem comedouros.

O teor de gordura visceral dos juvenis de tilápia do Nilo cultivados em tanques-rede com comedouros de formato quadrado e área de $100 \%$ apenas diferiu dos índices de gordura visceral dos peixes cultivados em tanques-rede sem comedouro. Navarro et al. (2010) não encontraram diferenças significativas para os dados de índice hepatossomático estudando suplementação de vitamina E em dietas para tilápia do Nilo. Da mesma forma, Bombardelli et al. (2009) e Bombardelli et al. (2010), avaliando o desempenho reprodutivo e zootécnico de fêmeas e de machos de tilápia do Nilo verificaram que níveis crescentes de energia digestível das rações também não influenciaram os índices he-

Tabela II.Valores (média e erro padrão -EP) para ganho em peso (GP), taxa de crescimento específico (TCE), sobrevivência (SOB) e conversão alimentar aparente (CAA), observados nos tanques-rede contendo diferentes formatos e áreas e ausentes de comedouros no cultivo de alevinos de tilápia do Nilo da linhagem GIFT (Values (mean and standard error -EP) for weight gain (GP), specific growth rate (TCE), survival (SOB) and feed conversion (CAA), observed in cages containing different shapes and areas and in the bsence of feeders in the cultivation of fingerlings of Nile tilapia, GIFT strain).

\begin{tabular}{|c|c|c|c|c|c|c|c|c|c|}
\hline & \multicolumn{2}{|c|}{ GP (g) } & \multicolumn{2}{|c|}{ TCE $(\%)$} & \multicolumn{2}{|c|}{ SOB $(\%)$} & \multicolumn{2}{|c|}{ CAA } \\
\hline & & Média & EP & Média & EP & Média & EP & Média & EP \\
\hline \multicolumn{2}{|l|}{ Formato } & \multicolumn{2}{|c|}{$F_{(1,16)}=1,0562 p=0,3194$} & \multicolumn{2}{|c|}{$F_{(1,16)}=0,044 p=0,9480$} & \multicolumn{2}{|c|}{$F_{(1,15)}=1,6013 p=0,2250$} & \multicolumn{2}{|c|}{$F_{(1,16)}=0,8500 p=0,3700$} \\
\hline \multicolumn{2}{|l|}{ Circular } & 35,62 & 0,54 & 3,14 & 0,03 & 97 & 0,00 & 1,26 & 0,02 \\
\hline \multicolumn{2}{|l|}{ Quadrado } & 36,35 & 0,47 & 3,14 & 0,04 & 97 & 0,00 & 1,24 & 0,02 \\
\hline \multicolumn{2}{|l|}{ Área (\%) } & \multicolumn{2}{|c|}{$F_{(3,16)}=0,1324 p=0,9394$} & \multicolumn{2}{|c|}{$F_{(3,16)}=0,4959 p=0,6903$} & \multicolumn{2}{|c|}{$F_{(3,15)}=4,3031 p=0,0223$} & \multicolumn{2}{|c|}{$F_{(3,16)}=0,2791 p=0,8397$} \\
\hline \multicolumn{2}{|l|}{25} & 36,12 & 0,77 & 3,14 & 0,03 & $97^{b}$ & 0,01 & 1,27 & 0,02 \\
\hline \multicolumn{2}{|l|}{50} & 36,29 & 1,19 & 3,16 & 0,07 & $98^{\mathrm{a}}$ & 0,00 & 1,24 & 0,02 \\
\hline \multicolumn{2}{|l|}{75} & 35,75 & 0,50 & 3,16 & 0,05 & $96^{b}$ & 0,01 & 1,25 & 0,03 \\
\hline \multicolumn{2}{|l|}{100} & 35,79 & 0,21 & 3,10 & 0,06 & $97^{\mathrm{ab}}$ & 0,00 & 1,25 & 0,01 \\
\hline Formato & Área (\%) & \multicolumn{2}{|c|}{$F_{(3,16)}=1,8171 p=0,1847$} & \multicolumn{2}{|c|}{$F_{(3,16)}=5,6109 p=0,0080$} & \multicolumn{2}{|c|}{$F_{(3,15)}=2,6365 p=0,0877$} & \multicolumn{2}{|c|}{$F_{(3,16)}=0,7806 p=0,5219$} \\
\hline Circular & 25 & $36,65^{\mathrm{A}}$ & 0,98 & $3,14^{\mathrm{abA}}$ & 0,07 & 97 & 0,01 & $1,26^{\mathrm{B}}$ & 0,02 \\
\hline Circular & 50 & $34,59^{A}$ & 1,98 & $3,05^{\mathrm{abB}}$ & 0,08 & 98 & 0,00 & $1,27^{\mathrm{B}}$ & 0,04 \\
\hline Circular & 75 & $35,39^{A}$ & 0,53 & $3,15^{\mathrm{abA}}$ & 0,09 & 94 & 0,02 & $1,28^{\mathrm{B}}$ & 0,05 \\
\hline Circular & 100 & $35,86^{A}$ & 0,24 & $3,23^{\mathrm{abA}}$ & 0,02 & 98 & 0,00 & $1,25^{\mathrm{B}}$ & 0,02 \\
\hline Quadrado & 25 & $35,59^{A}$ & 1,31 & $3,14^{\mathrm{abA}}$ & 0,02 & 96 & 0,01 & $1,28^{\mathrm{B}}$ & 0,04 \\
\hline Quadrado & 50 & $37,99^{A}$ & 0,47 & $3,27^{\mathrm{aA}}$ & 0,05 & 99 & 0,01 & $1,21^{\mathrm{B}}$ & 0,02 \\
\hline Quadrado & 75 & $36,12^{\mathrm{A}}$ & 0,90 & $3,17^{\mathrm{abA}}$ & 0,06 & 97 & 0,00 & $1,22^{\mathrm{B}}$ & 0,04 \\
\hline Quadrado & 100 & $35,72^{\mathrm{A}}$ & 0,40 & $2,97^{\mathrm{bB}}$ & 0,05 & 97 & 0,00 & $1,25^{\mathrm{B}}$ & 0,01 \\
\hline \multicolumn{2}{|c|}{ Controle, Isento } & $25,45^{\mathrm{B}}$ & 2,94 & $2,63^{\mathrm{B}}$ & 0,30 & 97 & 0,023 & $1,49^{A}$ & 0,05 \\
\hline \multicolumn{2}{|l|}{ CV (\%) } & 2,79 & & 3,05 & & 1,91 & & 1,91 & \\
\hline
\end{tabular}

Estatística F e p-valor são apresentados para cada fonte de variação. Coeficiente de variação (CV) entre os cultivos também é fornecido. *Letras minúsculas distintas nas colunas indicam diferenças significativas $(p<0,05)$ pelo teste de Tukey (comparação entre os cultivos) e letras maiúsculas distintas nas colunas indicam diferenças significativas $(p<0,05)$ pelo teste de Dunnett (comparação dos cultivos com o controle ou isento). 


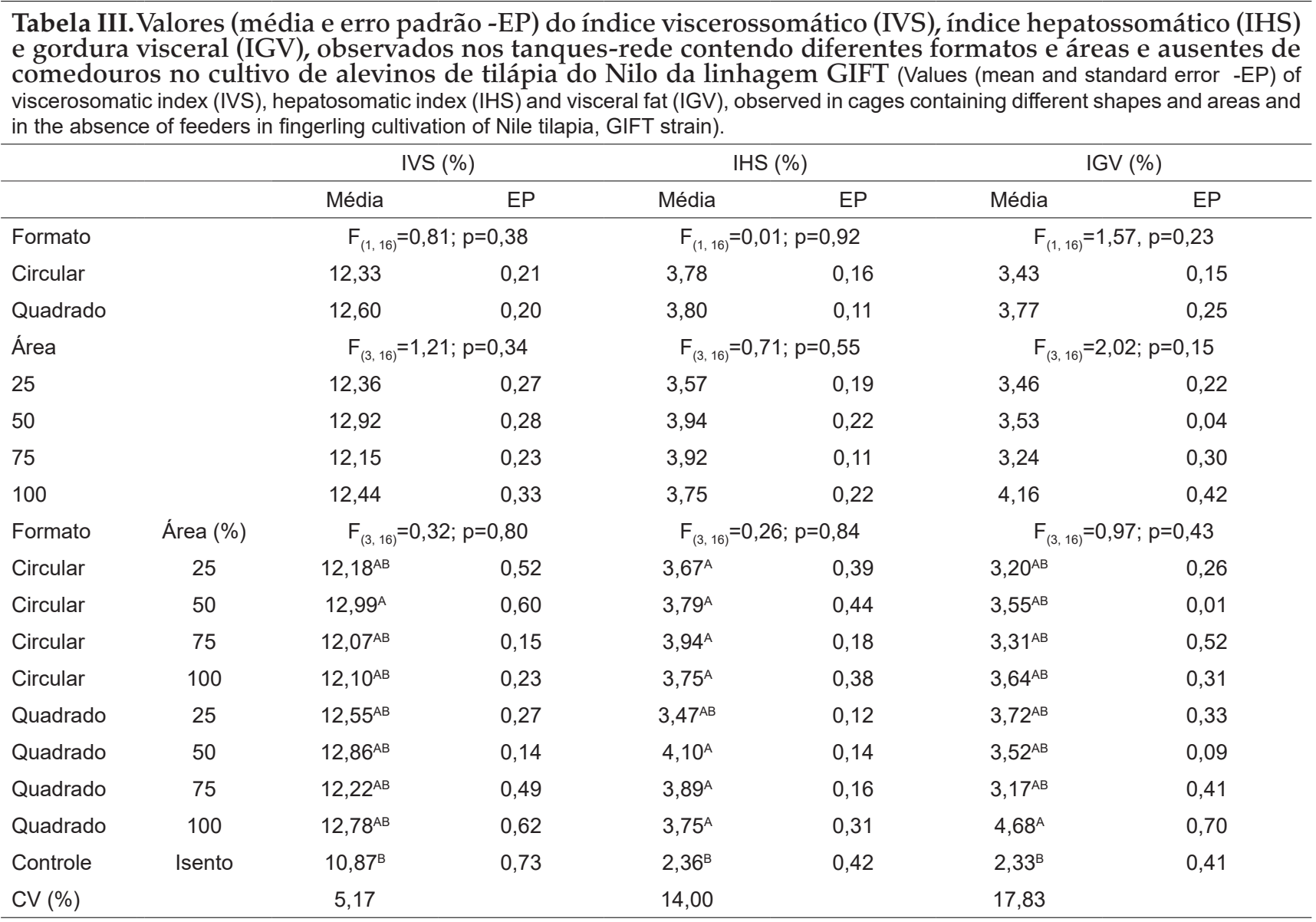

Estatística F e p-valor são apresentados para cada fonte de variação. Coeficiente de variação (CV) entre os cultivos também é fornecido. *Letras maiúsculas distintas nas colunas indicam diferenças significativas $(p<0,05)$ pelo teste de Dunnett (comparação dos cultivos com o controle ou isento).

patossomático, gonadossomático e viscerossomático dos animais.

Considerando que os peixes produzidos nos tanques-rede isentos de comedouros apresentaram menor desempenho zootécnico, possivelmente os resultados estejam relacionados com o desperdício de ração por falta do alimentador. Como a principal fonte de energia disponível são os lipídios, a má utilização de ração reforça a hipótese da menor quantidade de gordura visceral observada nos peixes destas unidades de cultivo. Os valores dos índices corporais avaliados podem também estar relacionados ao estresse do ambiente e competição pelo alimento, influenciando negativamente na fisiologia dos peixes (Rebouças et al., 2014).

Para os valores de desvio padrão obtidos do peso (DPPF), comprimento (DPCTF) e fator de condição (DPKn) dos lotes de criação de tilápias (tabela I) não foram verificados efeitos $(p>0,05)$ do formato, área e interação (formato e área). Estes resultados concordam com os encontrados por Marengoni et al. (2010). O formato e a área não proporcionaram efeito significativo para a variação testada, caracterizando uma homogeneidade dos lotes dos peixes com relação ao comprimento e peso médio final. Possivelmente este fato deve- -se as condições experimentais durante a fase de cultivo em que os peixes se encontravam. Diferentemente, Sandoval Jr. et al. (2008), descreveram a competição por alimento como um problema em comedouros circulares, resultando tal efeito em heterogeneidade de lote. Neste mesmo sentido, Neuman et al. (2009), avaliando o desempenho de três linhagens de tilápia do Nilo mantidas em condições ambientais não controladas, verificaram heterogeneidade no crescimento dos peixes em peso e crescimento total em função de canibalismo e variações de temperatura no decorrer dos 90 dias de cultivo.

Segundo Marengoni et al. (2010) as variâncias heterogêneas podem interferir no desempenho produtivo, no aspecto do crescimento heterogêneo, estabelecendo hierarquia entre os peixes maiores sobre os menores, resultando em desuniformidade do plantel. Desta forma, nas próximas fases de cultivo seriam necessários manejos intensos de seleções ou classificação das categorias de peso ou tamanho, tendo como consequência um menor lucro para o produtor. 
Portanto, faz-se necessário a utilização de comedouros em tanques-rede no cultivo de alevinos de tilápia do Nilo que podem ajudar a melhorar o processo de criação da espécie, mesmo que nas fases iniciais, neste sistema de criação. No entanto, é necessária a validação dos resultados em unidades comerciais com tanques-rede de pequeno volume e alta densidade de estocagem (Kubitza, 2011). A utilização de um dos dois formatos e áreas de comedouros durante o cultivo de juvenis em tanques-rede é importante para manter a padronização dos lotes, diminuindo a variação de tamanhos e pesos dos peixes. Além disso, a utilização desses aparatos no manejo de arraçoamento dos peixes contribui para aumentar a receita final para o piscicultor de forma sustentavelmente econômica, ambiental e social.

\section{CONCLUSÕES}

Os comedouros são indispensáveis no sistema de criação da tilápia do Nilo. As diferentes áreas e formatos de comedouros avaliados não interferem no desempenho produtivo na fase de juvenis de tilápia do Nilo em tanques-rede de pequeno volume. Recomenda-se a utilização de pelo menos uma das combinações entre área e formato de comedouro utilizado na fase cultivada.

\section{AGRADECIMENTOS}

Os autores agradecem à Fundação Araucária pela concessão de auxílio financeiro e ao Instituto de Pesquisa e Aquicultura Ambiental (InPAA) em Toledo, Paraná, Brasil pela infraestrutura disponibilizada para realização da pesquisa.

\section{BIBLIOGRAFIA}

Barroso, R.M.; Tenório, R.A.; Pedroza Filho, M.X.; Webber, D.C.; Belchior, L.S.; Tahim, E.F.; Carmo, F.J. e Muehlmann, L.D. 2015. Gerenciamento genético da tilápia nos cultivos comerciais. Embrapa Pesca e Aquicultura. Palmas. Brasil. 64 pp.

Bombardelli, R.A.; Hayashi, C.; Natali, M.R.M.; Sanches, E.A. ePiana, P.A. 2009. Desempenho reprodutivo e zootécnico e deposição de lipídios nos hepatócitos de fêmeas de tilápia-do-Nilo alimentadas com rações de diversos níveis energéticos. Rev Bras Zootecn, 38: 1391-1399.

Bombardelli, R.A.; Hayashi, C.; Natali, M.R.M.; Sanches, E.A. ePiana, P.A 2010. Níveis de energia digestível sobre os desempenhos reprodutivo e zootécnico e a deposição de lipídios nos hepatócitos de machos de tilápia-do-Nilo. Rev Bras Zootecn, 39: 941-949.

Boyd, C.E. 2005. Farm-level issues in aquaculture certification: tilapia. Report commissioned by world wild life - US. 29 pp.

Boyd, C.E. and Tucker, C.S. 1998. Pond aquaculture water quality management. Kluwer Academic Publishers. Boston. 700 pp.

Brasil. 2014. $1^{\circ}$ Anuário brasileiro da pesca e aquicultura. MPA. Brasília. 136 pp. (15/06/2017).

Cyrino, J.E.P.; Bicudo, A.J.A.; Sado, R.Y.; Borghesi, R. e Dairiki, J.K. 2010. A piscicultura e o ambiente-o uso de alimentos ambientalmente corretos em piscicultura. Rev Bras Zootecn, 39: 68-87.

El-Sayed, A.F.M. 2006. Tilapia culture. CABI Publishing. Cambridge. $277 \mathrm{pp}$.
FAO. 2016. The state of world fisheries and aquaculture 2016. Contributing to food security and nutrition for all. Rome. 200 pp.

Gomiero, L.M. and Braga, F.M.S. 2005. The condition factor of fishes from two river basins in São Paulo state, Southeast of Brazil. Acta Sci Biol Sci, 27: 73-78.

IBGE. 2015. Instituto Brasileiro de Geografia e Estatística. Produção Pecuária Municipal, 2014, v. 42.39 pp.

Kubitza, F. 2011. Tilápia: tecnologia e planejamento na produção comercial. 2 ed. Acqua Supre Com. Suprim. Aqüicultura Ltda. Jundiaí. $316 \mathrm{pp}$.

Manairdes-Pinto, C.S.R.; Paiva, P.; Verani, J.R.; Andrade-Talmelli, E.F.; Wirz, M.V.M.A. e Silva, A.L. 2007. Desempenho produtivo da tilápia tailandesa, Oreochromis niloticus, estocada em diferentes quantidades de tanques-rede instalados em viveiros povoados com a mesma espécie. Bol Inst Pesca, 33: 53-62.

Mamun, S.M.; Focken, U.; Francis, G. and Becker, K. 2004. Growth performance and metabolic rates of genetically improved and conventional strains of Nile tilapia, Oreochromis niloticus (L.), reared individually and fed ad libitum. In: Bolivar, R.; Mair, G.; Fitzsimmons, K. (Eds.). Proceedings of the Sixth International Symposium on Tilapia in Aquaculture. BFAR. Manila. Philippines. pp. 379-399.

Marengoni, N.G.; Bernadi, A. e Gonçalves Júnior, A.C. 2007. Tilapicultura vs. cultura da soja e do milho na região oeste do Paraná. Informações Econômicas, 37: 41-49.

Marengoni, N.G.; Possamai, M.; Gonçalves Júnior, A.C. e Oliveira, A.A.M.A. 2008. Performance e retenção de metais pesados em três linhagens de juvenis de tilápia-do-Nilo em hapas. Act Sci Anim Sci, 30: 351-358.

Marengoni, N.G.; Santos, R.S.; Gonçalves Junior, A.C.; Gino, D.M.; Zerbinatti, D.C.P. e Lima, F.S. 2009. Monogenoidea (Dactylogyridae) em tilápia do Nilo cultivada sob diferentes densidades de estocagem em tanques-rede. Arq Bras Med Vet Zootec, 61: 393-400.

Marengoni, N.G.; Albuquerque, D.M.; Mota, F.L.S.; Passos Neto, O.P.; Silva Neto, A.A.; Silva, A.I.M. e Ogawa, M. 2010. Desempenho produtivo de tilápia vermelha submetida à dieta contendo probiótico durante a alevinagem em água mesohalina. Arch Zootec, 59: 403-414. Merighe, G.K.F.; Pereira-da-Silva, E.M.; Negrão, J.A. e Ribeiro, S. 2004. Efeito da cor do ambiente sobre o estresse social em tilápias do Nilo (Oreochromis niloticus). Rev Bras Zooten, 33: 828-837.

Moraes, A.M.; Seiffert, W.Q.; Tavares, F. e Fracalossi, D.M. 2009. Desempenho zootécnico de tilápia do Nilo, Oreochromis niloticus, em tanques-rede, com diferentes rações comerciais. Rev Ciênc Agron, 40: 388-395.

Navarro, R.D.; Ferreira, W.M.; Ribeiro Filho, O.P.; Veloso, D.P.; Fontes, D.O. e Silva, R.F. 2010. Desempenho de tilápia do Nilo/Oreochromis niloticus) suplementada com vitamina E. Arch Zootec, 59: 185-194.

Neumann, E.; Koberstein, T.C.R.D. e Braga, F.M.S. 2009. Desempenho de três linhagens de tilápia submetidas ao tratamento com 17-a-metiltestosterona em condições ambientais não controladas. Rev Bras Zootecn, 38: 973-979.

Neves, P.R.; Ribeiro, R.P.; Vargas, L.; Natali, M.R.M.; Maehana, K.R. and Marengoni, N.G. 2008. Evaluation of the performance of the two strains of Nile tilapia (Oreochromis niloticus) in mixed raising systems. Braz Arch Biol Technol, 51: 531-538.

Rebouças, P.M.; Rocha, R.S.; Silva, M.C.; Barbosa Filho, J.A.D.; Farias, W.R.L.; Pinto, C.R.S. and Henrique, J.C. 2014. Influence of environmental color on zootechnical performance and feeding behavior during masculinization of Nile tilapia. J Anim Behav Biometeorol, 2: 126-130.

Rezk, M.A.; Kamel, E.A.; Ramadan, A.A. and Dunham, R.A. 2002. Comparative growth of Egyptian tilapias in response to declining water temperature. Aquaculture, 207: 239-247.

Rodrigues, P.C. 2002. Bioestatística. EdUFF. Niterói. Río de Janeiro. Brasil. 339 pp.

Sandoval Junior, P.; Trombeta, T.D. e Mattos, B.O. 2013. Manual de criação de peixes em tanque-rede. 2. ed. Codevasf. Brasília. 68 pp. 
Siangas, L.E; Pouilly, M.; Vallejos, A.; Pérez, T. and Rejas, D. 2012. Effect of water quality on growth of four fish species in the lténez basin (Upper Madera, Amazon). Env Biol Fish, 95: 371-381.

Tachibana, L.; Leonardo, A.F.G.; Corrêa, C.F. eSaes, L.A. 2008. Densidade de estocagem de pós-larvas de tilápia-do-Nilo (Oreochromis niloticus) durante a fase de reversão sexual. Bol Inst Pesca, 34: 483-488.

Tavares-Dias, M.; Martins, M.L. e Moraes, F.R. 2000. Relação hepatossomática e esplenossomática em peixes teleósteos de cultivo intensivo. Rev Bras de Zool, 17: 273-281.
Tavares-Dias, M.; Marcon, J.L.; Lemos, J.R.G.; FIM, J.D.I.; Affonso, E.G. e Ono, E.A. 2008. Índices de condição corporal em juvenis de Brycon amazonicus (Spix and Agassis, 1829) e Colossoma macropomum (Cuvier, 1818) na Amazônia. Bol Inst Pesca, 34: 197-204.

Wild, M.B.; Marengoni, N.G.; Vivian, M.M.P.S.; Tsutsumi, C.Y. e Moura, M.C. 2014. Probiótico dietético em sistemas de produção de tilápia do Nilo: efeitos sobre o crescimento, balanço de $\mathrm{N}$ e $\mathrm{P}$, retenção de nutrientes e viabilidade econômica. Semin Cienc Agrar, 35: 477-490. 